\title{
COTS FPGA/SRAM Irradiations Using a Dedicated Testing Infrastructure for Characterization of Large Component Batches
}

Slawosz Uznanski, Benjamin Todd, Johannes Walter, Andrea Vilar-Villanueva

CERN, Geneva, Switzerland

Keywords: Total Ioninzing Dose (TID), Single Event Effects (SEE), Commercial-Off-The-Shelf (COTS) components, Static Random Access Memory (SRAM), Field Programmable Gate Array (FPGA)

\begin{abstract}
This paper introduces a new testing platform for irradiation of large batches of COTS FPGA and SRAMs. The main objective is measurement of component radiation response and assessment of component-to-component variability within one batch. The first validation and test results using the testing platform are presented for 150nm TFT SRAM (Renesas) and different sizes of the 130nm ProASIC3 FPGA (Microsemi).
\end{abstract}

Presented at: MIXDES 2014, 19-21 June 2014, Lublin, Poland

Geneva, Switzerland

January, 2015 


\title{
COTS FPGA/SRAM Irradiations Using a Dedicated Testing Infrastructure for Characterization of Large Component Batches
}

\author{
Slawosz Uznanski, Benjamin Todd, Johannes Walter, Andrea Vilar-Villanueva \\ Technology Department, Electric Power Converters, Converter Controls Electronics, (TE-EPC-CCE) \\ European Organization for Nuclear Research (CERN) \\ Route de Meyrin 385, 1217 Meyrin, Switzerland \\ E-mail: slawosz.uznanski@cern.ch, Office: +41 227662591
}

\begin{abstract}
This paper introduces a new testing platform for irradiation of large batches of COTS FPGA and SRAMs. The main objective is measurement of component radiation response and assessment of component-to-component variability within one batch. The first validation and test results using the testing platform are presented for 150nm TFT SRAM (Renesas) and different sizes of the 130nm ProASIC3 FPGA (Microsemi).
\end{abstract}

Keywords-Total Ioninzing Dose (TID), Single Event Effects (SEE), Commercial-Off-The-Shelf (COTS) components, Static Random Access Memory (SRAM), Field Programmable Gate Array (FPGA)

\section{INTRODUCTION}

A new power converter control system for the Large Hadron Collider (LHC) at the European Organization for Nuclear Research (CERN) is currently under development and will be based on custom-designed electronic systems called Function Generator Controllers (FGCs) [1]. These FGCs will embed Commercial-Off-The-Shelf (COTS) components, which will be exposed to substantial high energy particle fluxes [2] inducing Single Event Effects (SEE) and Total Ionizing Dose (TID) degradation. At the system level, these can translate to a premature end to physics operation (a beam dump) and therefore have an impact on the availability of the LHC machine.

Cost and availability from different manufacturers have propelled the use of COTS in different radiation environments [3-4]. Each single integrated circuit needs to be qualified for a given environment to estimate its radiation response. However, the main challenge is to assess component-to-component variability of radiation response within a single batch and estimate the error rate in the final operating environment. In addition, die traceability during manufacturing (silicon lot date code, position on wafer or packaging date code) is very often difficult if not impossible to obtain from distributors. In the case of the LHC power converter controls, an additional challenge is the large number of systems required, thus very low error rates are needed for the individual components. Component testing is therefore a crucial element of the design flow and requires a complete testing infrastructure to prove designs meet their targets.

The main objective of this work is to present the architecture of a testing platform to be used in radiation facilities to validate batches of two types of semiconductors for their use in the LHC: Static Random Access Memories (SRAM) and Field Programmable Gate Arrays (FPGA). This paper is organized as follows: Section II presents the Testing Platform. Section III presents results from radiation campaigns of SRAMs and FPGAs. The future work is presented in the final section.

\section{BATCH TESTING PlATFORM}

\section{A. Specification}

The objective is to be able to fully characterize the radiation response of commercial SRAMs and FPGAs, i.e. the SEE cross-section (mainly Single Event Upset - SEU and Single Event Latch-up - SEL) and the TID degradation. For this purpose, a constant power monitoring with fast overcurrent detection and a power cycle circuit needs to be implemented as well as a possibility to access to all component functional data.

In addition, the platform should allow simultaneous testing of multiple components to characterise sensitivity-variability within a large batch while limiting the beam time required. The hardware must be modular, easy to reuse/debug and adaptable to different DUTs and radiation facilities. The tests are planned at three main facilities: PIF at PSI [5] in Villigen in Switzerland, PIF at TRIUMF [6] in Vancouver in Canada and CHARM at CERN [7].

\section{B. Overview of the architecture}

Fig 1 presents the block diagram of the system which is composed of three main parts: 1) Target area in which the DUT boards are placed on the Test Control Card (TCC), 2) Low radiation area in which the control electronics is placed that stimulates DUTs, collects outputs and performs measurements needed for a full characterization and 3) Control room in which a supervision equipment is connected to the control electronics. 


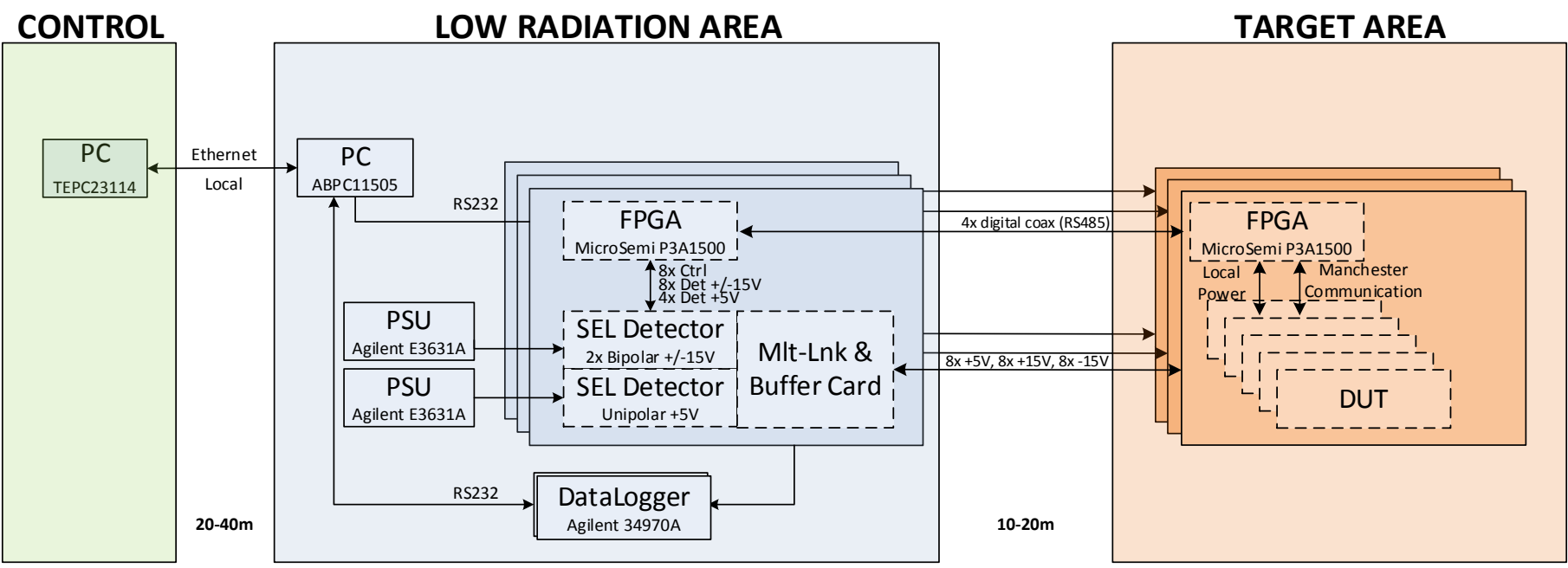

Fig. 1. Block diagram of the batch testing infrastructure is composed of three main parts: 1) Up to 8x Device Under Test (DUT) and Test Control Card (TCC) boards placed in the radiation beam (Target Area), 2) Control electronics placed on the long (12m) cables behind shielding (Low Radiation Area), and 3) Supervision equipment placed in a safe location to which test operators have access during irradiations (Control).

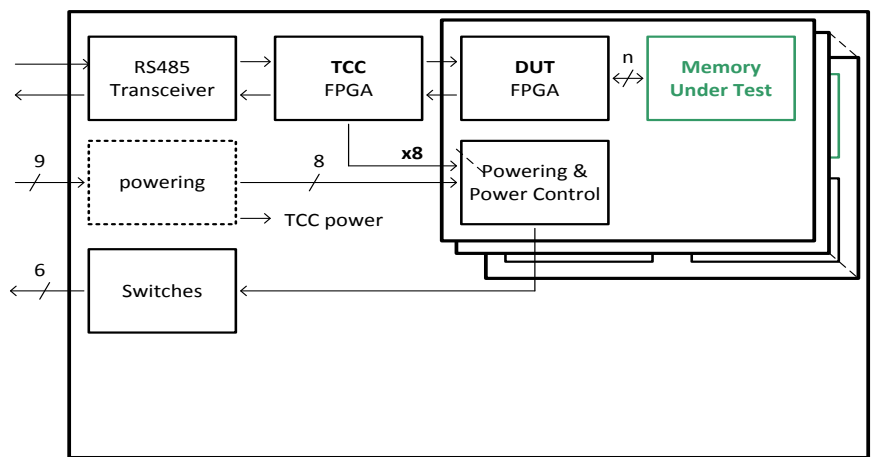

Fig. 2. Block diagram of the Test Control Card (TCC) and the Device Under Test (DUT) board. The TCC FGPA implements high throughput communication with the DUT FPGA, while communication with controls implements a low throughput communication over the RS485 link.

\section{Controls part}

A PC or an industrial computer is connected to an Analogue Data Acquisition Unit (Agilent 34970A) for all slow analogue power consumption measurements needed to compute the TID degradation. Additionally, the same computer is connected to a dedicated full-custom electronic module embedding an FPGA which allows a fast digital data acquisition from the TCC and controls the overcurrent events (SEL detector). The overcurrent detection time constant can be adjusted between several hundreds of microseconds to seconds and the detection thresholds between several milliamps to several hundreds of milliamps. After each SEL, a power cycle is performed to restore a correct function and protect the DUT from thermal failure. Power is delivered from Power Supply Units (Agilent E3631A).

\section{TCC and DUT part}

Fig. 2 shows a block diagram of the Test Control Card (TCC) and DUT boards. On one side, the TCC FGPA is connected to the controls electronics via a dedicated low throughput RS485 communication, power lines and switches to control all analogue measurements. On the other side, the TCC
FPGA stimulates the DUT boards with a correct input data and collects the digital output information for data analysis using a high throughput direct link.

Each DUT board embeds its own powering circuit, a FPGA and an SRAM memory (if needed). There are two reasons for this implementation: 1) while testing SRAMs, a FPGA is needed to collect and analyse the large amount of data from the SRAM and 2) Implementing both components on one DUT board allows both to be tested using a single platform, thus decreasing cost.

An additional study was performed in order to optimize component placement in the beam. It was decided to expose up to 8 stacked boards in the beam line one behind another. An SRIM simulation [8] has been performed on a simple model and it was found out that the beam attenuation between the first board (upstream) and the $8^{\text {th }}$ one (downstream) is lower than $5 \%$ for the mono-energetic $230 \mathrm{MeV}$ proton beam of PSI.

\section{FIRST IRRADIATION CAMPAIGNS}

\section{A. PSI and TRIUMF facilities}

The test setup was validated in the PIF facilities at PSI and at TRIUMF. In both facilities, a mono-energetic proton beam between several to several hundreds of $\mathrm{MeV}$ is available. Tests were performed in compliance with the ESA/SCC Basic Specification No. 25100 [9] with the following energies: 60, 100, 230, 350 and $480 \mathrm{MeV}$.

\section{B. Radiation flux mesurements for different stacked boards}

A dedicated measurement was done at PSI to assess particle flux variations between board positions in order to be able to account for particle flux variations between different boards. Table 1 summarizes these results. As predicted for the highest energy of $230 \mathrm{MeV}$, the difference between the upstream $(5 \mathrm{~cm}$ from collimator) and downstream (16 cm from collimator) boards is negligible. When beam energy is lowered using dedicated copper scatterers at 100 and $60 \mathrm{MeV}$, this difference becomes significant (up to $40 \%$ ) and needs to be accounted for 
by data analysis. This difference is due to the presence of the scatterer that spreads the beam and causes the flux variations as a function of distance from the scatterer.

TABLE I. MEASUREMENTS OF THE PROTON FLUX AND THE DOSE RATE IN SILICON AS A FUNCTION OF ENERGY AND A DISTANCE FROM COLLIMATOR: 5CM FROM COLLIMATOR CORRESPONDS TO THE UPSTREAM BOARD POSITION AND 16CM FROM COLLIMATORS TO THE DOWNSTREAM BOARD.

\begin{tabular}{|c|c|c|c|c|}
\hline $\begin{array}{c}\text { Energy } \\
(\mathrm{MeV})\end{array}$ & $\begin{array}{c}\text { Distance from } \\
\text { collimator }(\mathbf{c m})\end{array}$ & $\begin{array}{c}\text { Flux } \\
(\mathbf{p} / \mathbf{c m} 2 / \mathbf{s})\end{array}$ & $\begin{array}{c}\text { DUT } \\
\text { position }\end{array}$ & $\begin{array}{c}\text { Dose Rate } \\
\text { (Gy/s) }\end{array}$ \\
\hline \multirow{2}{*}{230} & 5 & $1.74 \mathrm{E}+08$ & 1 & $9.2 \mathrm{E}-02$ \\
\cline { 2 - 5 } & 16 & $1.74 \mathrm{E}+08$ & 8 & $9.2 \mathrm{E}-02$ \\
\hline \multirow{2}{*}{100} & 5 & $1.02 \mathrm{E}+08$ & 1 & $9.5 \mathrm{E}-02$ \\
\cline { 2 - 5 } & 16 & $7.00 \mathrm{E}+07$ & 8 & $6.5 \mathrm{E}-02$ \\
\hline \multirow{3}{*}{60} & 5 & $7.55 \mathrm{E}+07$ & 1 & $1.1 \mathrm{E}-01$ \\
\cline { 2 - 5 } & 7 & $7.10 \mathrm{E}+07$ & 3 & $9.7 \mathrm{E}-02$ \\
\cline { 2 - 5 } & 16 & $4.40 \mathrm{E}+07$ & 8 & $6.0 \mathrm{E}-02$ \\
\hline
\end{tabular}

\section{Renesas memory test results}

The SRAM memory (Renesas Corp.) was manufactured in a 150nm process using a Thin Film Transistor (TFT) PMOS technology enhanced with Dynamic Random Access Memory (DRAM) capacitors [10]. This makes this memory very promising for use in radiation environments due to lack of the NPNP thyristor inherent to CMOS structures, thus meaning the memory is SEL-immune. The addition of a Dynamic Random Access Memory (DRAM) capacitor decreases the SEU crosssection. Two different component batches were purchased from Renesas Corp: Date Code 1343 (MSC batch 2013430001), and 1328 (MSC batch 2013280004).

The expected immunity for SEL was confirmed during both campaigns. Fig 3 shows the installed test setup in the target area at TRIUMF while Fig. 4, shows the measured SEU crosssection as a function of energy. The extremely low SEU sensitivity ( $\sim 3$ orders of magnitude lower than standard SRAMs) measured previously by NASA [11] was confirmed. The small discrepancy is likely due to differences in manufacturing processes between 2007 and 2013. A detailed analysis of the test results can be found in [12].

A total of 21 different memories were irradiated in both facilities. To analyse the variation of the SEU cross-section between different chips, the $230 \mathrm{MeV}$ data were used for which the best statistics was obtained on 10 different chips coming from two purchased batches. Data are presented in Fig. 5. The ratio between the lowest and highest measured value is equal to 2.3x, while between maximum 90\% confidence upper level and minimum $90 \%$ confidence level of the SEU cross-section is as high as $3.7 \mathrm{x}$. This result shows the importance of response variation between different chips in estimating the error rate in the final application. Interestingly for these components, both maximum and minimum SEU cross-section values are observed within one batch.

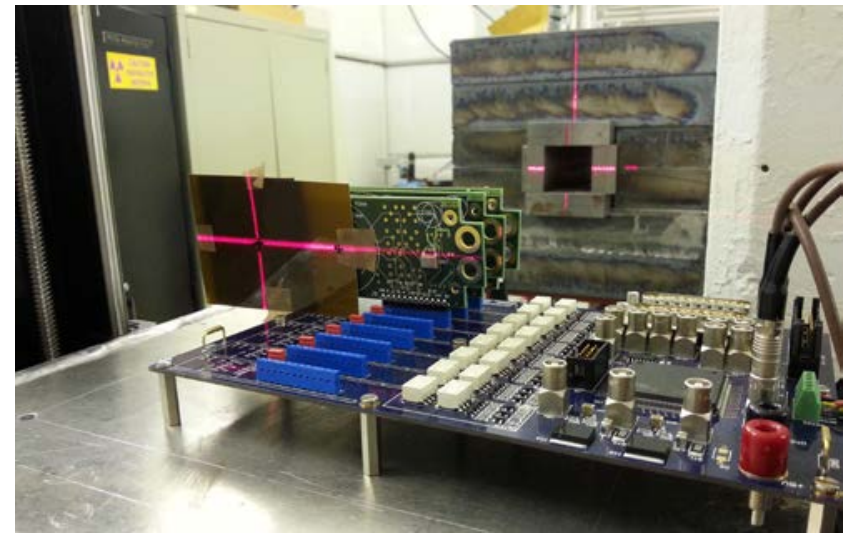

Fig. 3. A Test Control partially populated with DUT boards during the Renesas SRAM memory tests performed at PIF facility at TRIUMF. The laser pointers show the alignment of the DUT components with respect to the beam. A gafchromic film was used in order to cross-check the relative position of the beam/DUT components and to verify collimation system.

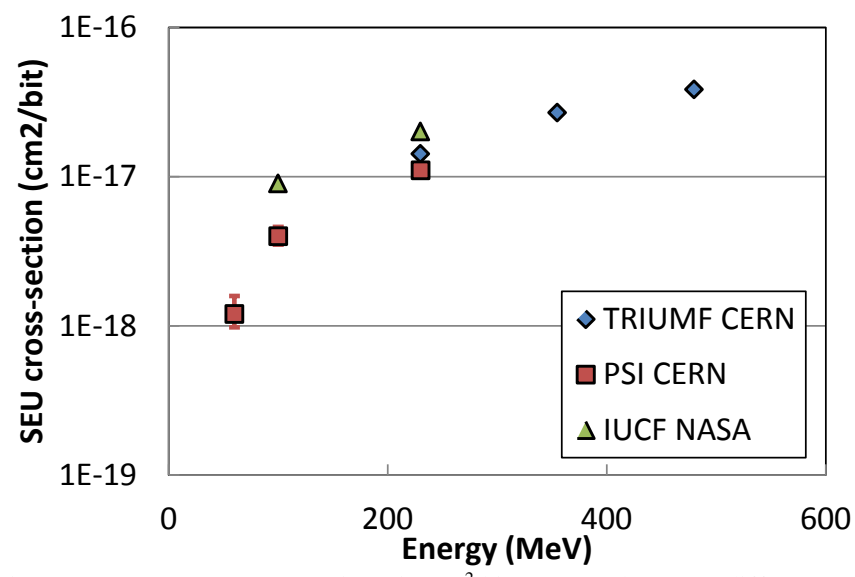

Fig. 4. Proton SEU cross-sections in $\mathrm{cm}^{2} /$ bit measured at two different test facilities: PSI (Oct 2013), TRIUMF Dec 2013 and compared to data collected by MEI Technologies/NASA at IUCF (Jun 2007) [9]. The error bars (90\% confidence level) are shown only for CERN irradiations when relevant.

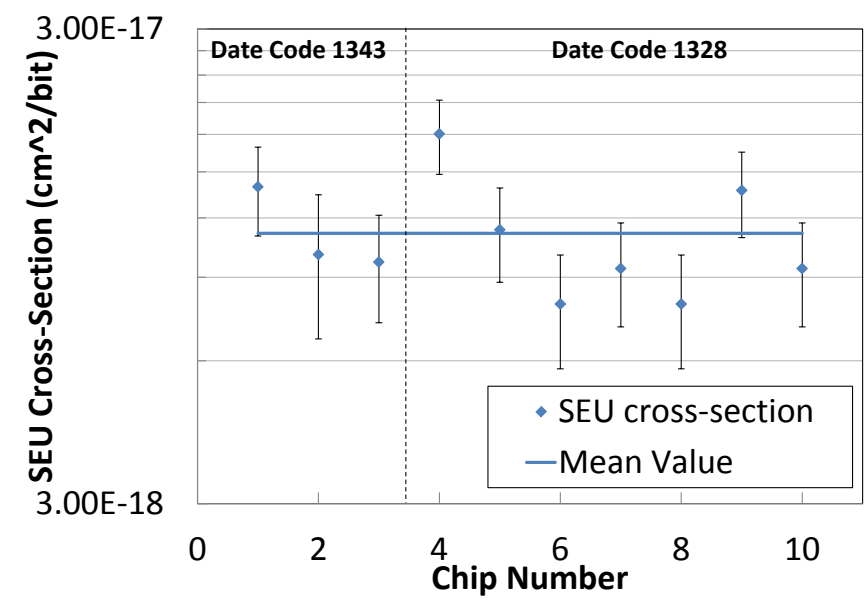

Fig. 5. Proton SEU cross-section for different chips irradiated at $230 \mathrm{MeV}$. The $90 \%$ confidence upper and lower level were plotted.

\section{Microsemi FPGA test results}

The aim of the FPGA test campaign was to measure the SEU cross-section of Random Access Memory (RAM) blocks 
and Flip-Flops (FF) across different sizes of the ProASIC FPGAs. These cross-section data have already been published before for the biggest A3PE3000L [13] Nevertheless, the differences of cross-sections as a function of device size have not been documented.

Different component samples were purchased: A3P250 (PQ208), A3P400 (FG484), A3P1000 (FG484) and A3PE1500 (FG484). Two different test designs were prepared: 1) instantiating all available RAM blocks in each FPGA and 2) instantiating a big shift register using $90 \%$ of all FF of each FPGA. Fig. 6 shows the installed test setup at PSI ready for FF SEU measurements. Fig 7 summarizes the measurement data showing previously published results [13] for comparison purposes. The results collected by CERN and by ESA/Hirex irradiations are in good agreement with the exception of the RAM measurement at $60 \mathrm{MeV}$. The reason for this discrepancy has not been found. The differences between RAM and FF cross-section values for different FPGA sizes are moderate and will be presented in the final paper.

When considering the FF SEU cross-section as a function of the component, the variations for the same component size are limited to $5 \%$ while the differences between device sizes are within $20 \%$. The respective values for RAM variations are $10 \%$ for the same component size and 30\% between device sizes.

\section{FUTURE WORK}

The next radiation campaigns are scheduled in order to validate parts of firmware for the new power converter controls of LHC. The DUT boards can embed different sizes of FPGAs and up to 8 components can be irradiated at the same time, thus giving a possibility to simultaneously irradiate over 300k FFs and over 2Mbits of SRAMs. The testing infrastructure presented in this paper is going to be used for irradiations of the complex digital designs. As an example, a design prepared for the next test campaign consists of two interleaved filter designs with a generation of the coefficients on the fly used to reconstruct the digitized data from a 22-bit high precision delta-sigma ADC can be instantiated 176 times thus giving a possibility to obtain a cross-section resolution of $7.9 \times 10^{-15} \mathrm{~cm}^{2}$ per instance per hour of beam time. This shows a possibility to obtain an extreme event statistics per unit of time, not possible while irradiating one design. The targeted SEE cross-section upper limits in the range of $10^{-13}$ to $10^{-12} \mathrm{~cm} 2 /$ instance can be obtained in minutes.

\section{CONCLUSIONS}

This paper presents the architecture and initial test results from a testing platform, which allows irradiation different FPGAs and SRAMs. A large number of components can be irradiated at the same time in order to be able to decrease the beam time and also to measure the component-to-component variability of a radiation response.

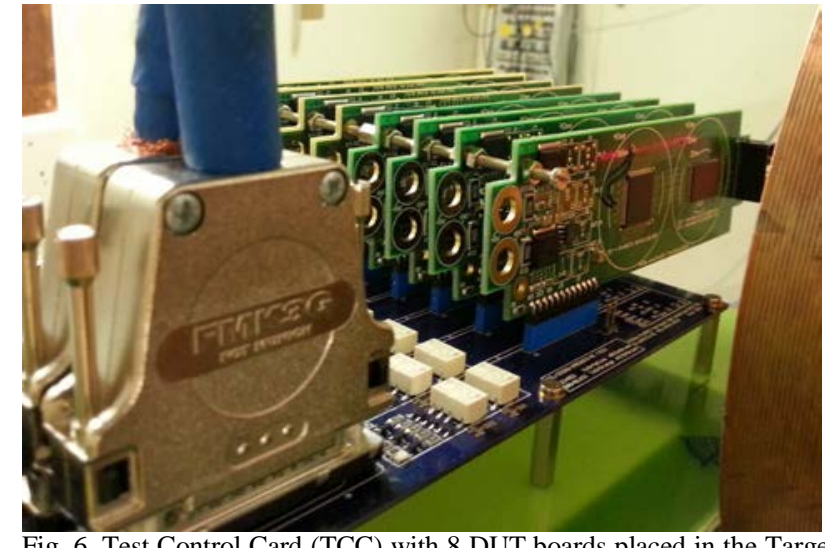

Fig. 6. Test Control Card (TCC) with 8 DUT boards placed in the Target Area of the PIF Facility at PSI during the FPGA irradiations. On the right the end of the beam collimator is visible; on the left the part of the beam dump.

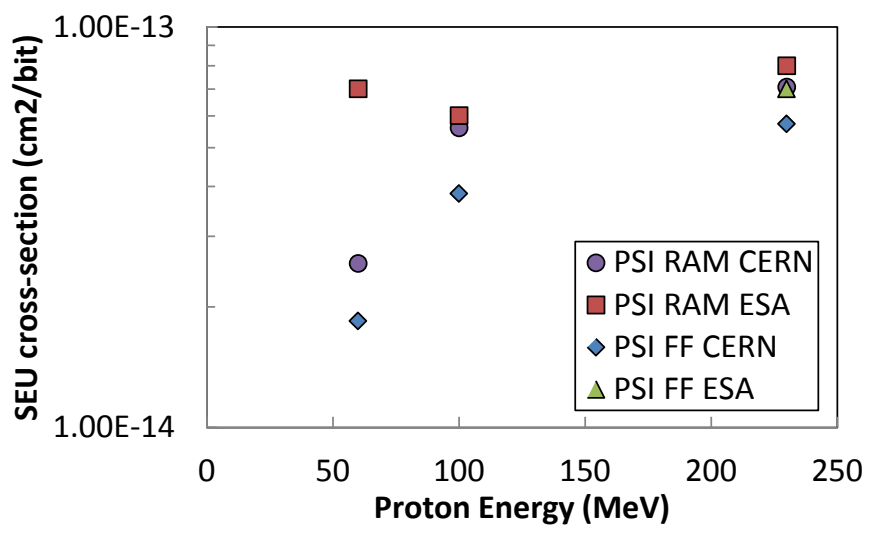

Fig. 7. Proton SEU cross-sections in $\mathrm{cm}^{2} /$ bit measured at PSI (Oct 2013), and compared to data collected by Hirex/ESA (Aug 2011) [13].

\section{REFERENCES}

[1] S. Uznanski, et al. "Radiation Hardness Assurance Methodology of Radiation Tolerant Power Converter Controls for LHC,” NSS 2013.

[2] K. Roed et al., "FLUKA simulations for SEE studies of critical LHC underground areas,” IEEE Tran. Nucl. Sci., June 2011.

[3] K.A. LaBelat al., "Emerging radiation hardness assurance (RHA) issues: a NASA approach for space flight programs,” Trans. Nucl. Sci., 1996.

[4] F. Irom, et al., "Compendium of SEL and TID Test Results of Commercial Analog to Digital Converters,” REDW, 2012.

[5] W. Hajdas, et al., "Radiation effects testing facilities in PSI during implementation of the proscan project," in REDW, 2002.

[6] E. Blackmore, "Operation of the triumf (20-500 mev) proton irradiation facility,” in Radiation Effects Data Workshop, 2000, 2000, pp. 1 -5.

[7] Available online: cern.ch/charm.

[8] J. F. Ziegler, "Stopping of energetic light ions in elemental matter," Journal of Applied Physics, 1999.

[9] European Space Agency, "Single Event Effects Test Mothod and Guidelines,”ESA/SCC Basic Specification No. 25100.

[10] Renesas R1LV1616R Datasheet, Renesas Electronics Corp, Apr 2010.

[11] S. Mackey, et al., "SEE Test Report V1.0 Heavy Ion SEE Test of R1LV1616RBG-7SI LPSRAM from Renesas,” Jun 2007.

[12] S. Uznanski, et al., "The Effect of Proton Energy on SEU Cross-Section of a 16Mbit TFT PMOS SRAM with DRAM Capacitors,” Submitted to the 2014 Nuclear and Space Radiation Effects Conference.

[13] M. Grandjean, et al., "Single Event Effects Test Report A3PE3000L," Aug. 2011. 